ability of Opil, they found that Opil was activated and translocated to the nucleus in the absence of inositol.

So, this work has clarified the details of a negative-feedback loop that controls phospholipid metabolism in yeast. The ER pool of PA directly binds to Opil to maintain it in an inactive state, but in the presence of inositol, this pool is consumed. The decreasing PA levels result in the release of Opil from the ER and its translocation to the nucleus, where it can repress the synthesis of inositol. Although many proteins are known to bind $\mathrm{PA}$, the physiological significance of this has been unclear. This study, which has shown a physiological response to changing PA levels, therefore indicates that specific pools of PA might have important signalling roles in other cells.

Rachel Smallridge

(i) References and links ORIGINAL RESEARCH PAPER Loewen, C. J. A. et al. Phospholipid metabolism regulated by a transcription factor sensing phosphatidic acid. Science 304, 1644-1647 (2004)

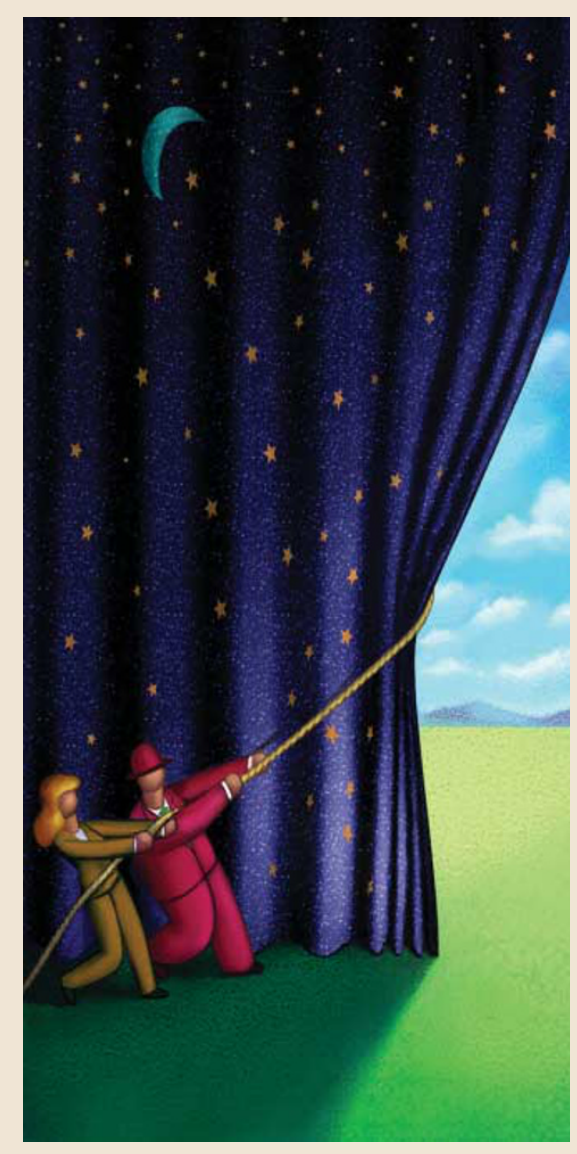

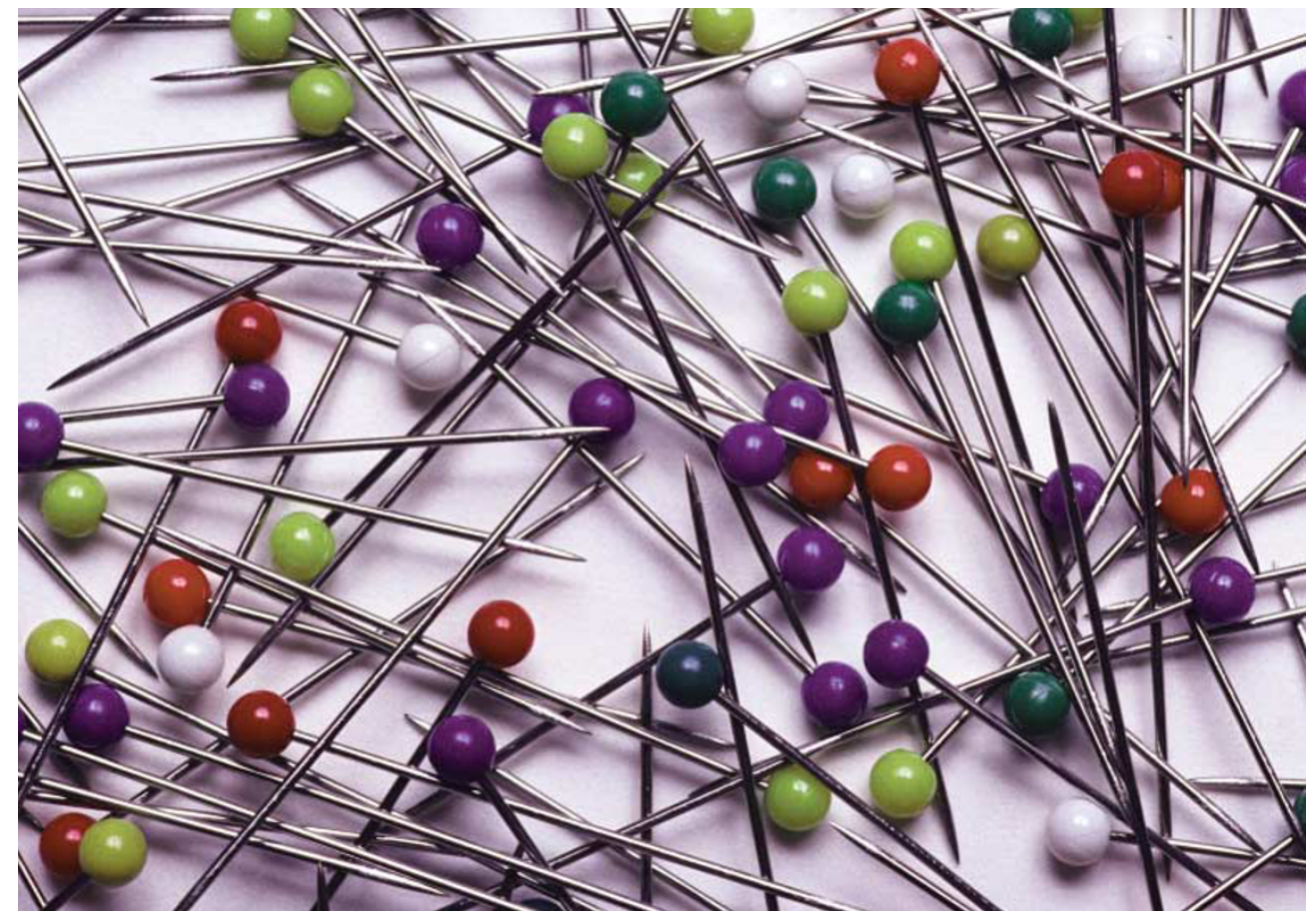

TUMOUR SUPPRESSORS

\section{Pinning down p73}

Two recent studies, one in Molecular Cell and the other in The Journal of Experimental Medicine, have shed new light on the molecular mechanisms that regulate the function and stability of p73 - a close relative of the tumour suppressor $\mathrm{p} 53$.

In the first study, Giannino Del Sal and colleagues investigated whether the prolyl isomerase Pin1, which is involved in regulating the apoptotic function of p53, has a similar effect on p73 function. This turned out to be the case, as Pin 1 strongly enhanced p73-dependent apoptosis in p53-null cells. This effect correlated with the Pin1-dependent induction of pro-apoptotic 73 target genes, including PUMA and $\mathrm{p} 53^{\mathrm{AIP} 1}$.

Pin1 recognizes phosphorylated serine or threonine residues followed by proline (pSer/pThr-Pro; where $\mathrm{p}$ denotes phosphorylation), and Pin1 binding induces a conformational change in its substrate. Mutational analysis of Ser/Thr-Pro sites in p73 identified three residues in the carboxyl terminus that, when mutated, decreased the Pin1-binding ability and the transcriptional activity of p73. The authors showed a Pin1-dependent conformational change in p73 in vitro, and found that Pin1-modified $\mathrm{p} 73$ has an increased affinity for the acetyl transferase p300. The Pin1-induced conformational change caused the p300-mediated acetylation of p73, which, in turn, promoted the transcriptional activation of $\mathrm{p} 73$ gene targets.

Pin 1 binding to 73 also increased the half-life of p73, and the absence of Pin 1 destabilized its substrate, both in normal and genotoxic-stress conditions. p73 is activated and stabilized in response to genotoxic stress by the Abl tyrosine kinase, through the $\mathrm{p} 38$ mitogen-activated protein kinase (MAPK) pathway. Del Sal and co-workers showed that the overexpression of Abl or p38 increased Pin 1-p73 binding in response to genotoxic stress. And, importantly, the Abl-mediated stabilization of p73 and the activation of its apoptotic function were dependent on the presence of Pin 1. So, the Del Sal group concluded that Pin 1 is essential for the activation of the apoptotic response by $\mathrm{p} 73$, and that, given its role in the functioning of p53, Pin 1 might be a common regulator of the p53 family.

Pier Paolo Pandolfi and co-workers also studied p73 stability, although from a different angle. The degradation of p53 is regulated by the ubiquitin-proteasome pathway, and the Pandolfi group showed that the same pathway is responsible for p 73 degradation. They then made an interesting observation - the overexpression of the tumour suppressor promyelocytic leukaemia (PML) caused the dose-dependent accumulation of $\mathrm{p} 73$, which coincided with reduced levels of ubiquitylated p73.

So, PML seems to protect $\mathrm{p} 73$ from ubiquitylation, but how? PML is known to promote the acetylation of $\mathrm{p} 53$ by CREB-binding protein (CBP), and Pandolfi and colleagues showed that PML has a similar effect on p73 - it causes the p300-mediated acetylation and stabilization of $\mathrm{p} 73$. The authors suggest that the competition between acetylation and ubiquitylation might be responsible for regulating the steady-state levels of p73, which represents a new mechanism for the regulation of protein stability.

Given the involvement of PML in the pathogenesis of acute promyelocytic leukemia (APL), p73 might also be implicated in this disease. The authors hypothesize that the stability and activity of p73 might be compromised in APL. Finally, the findings of these studies make one wonder how p63 stability and function are regulated..

Arianne Heinrichs

\section{(2) References and links}

ORIGINAL ReSEARCH PAPERS Mantovani, F. et al. Pin 1 links the activities of C-Abl and p300 in regulating p73 function. Mol. Cell $\mathbf{1 4}$

625-636 (2004) | Bernassola, F. et al. Ubiquitin-dependent degradation of p73 is inhibited by PML. J. Exp. Med. 199, 1545-1557 (2004) FURTHER READING Urist, M. \& Prives, C. The linchpin? Pin1 meets p73. Cancer Cell 5, 515-517 (2004) | Bernardi, R. et al. PML regulates p53 stability by sequestering Mdm2 to the nucleolus. Nature Cell Biol. 6, 665-672 (2004) 